\title{
Common fixed point theorems in intuitionistic fuzzy metric spaces and intuitionistic $(\phi, \psi)$-contractive mappings
}

\author{
H. M. Abu-Donia ${ }^{a}, *$, H. A. Atia ${ }^{a}$, Omnia M. A. Khater \\ ${ }^{a}$ Department of Mathematics, Faculty of Science, Zagazig University, Zagazig, Egypt. \\ ${ }^{b}$ Department of Basic Science, Zagazig Higher Institute of Engineering and Technology, Egypt.
}

\begin{abstract}
In this paper, we introduced the concept of intuitionistic $(\phi, \psi)$-contractive mappings and proved some a common fixed point theorems in intuitionistic fuzzy metric space under $(\phi, \psi)$-contractive mappings and weakly commuting intuitionistic fuzzy metric space.
\end{abstract}

Keywords: Intuitionistic fuzzy metric space, $(\phi, \psi)$-contractive mapping, weakly commuting, fixed point. 2010 MSC: $47 \mathrm{H} 10,54 \mathrm{H} 25$.

(C)2020 All rights reserved.

\section{Introduction}

In 1965, some author introduced the notion of the concept of fuzzy set theory which is one of the vital and useful branch of mathematics [12]. In 1986, the notion of an intuitionistic fuzzy set was introduced by Atanassov [2]. Kramosil and Michalek presented the definition of fuzzy metric space [5]. Many authors have studied fuzzy metric spaces (cf. [3, 11, 13]). In 2004, Park studied the concept of intuitionistic fuzzy metric space with $*$ continuous t-norm and $\diamond$ continuous t-conorm [7]. A generalization of commutativity was introduced by Sessa [8]. Common fixed point theorem was proved by Jungck [4] and introduced the concept of weakly compatible. Fuzzy $\psi$-contractive mappings in non-Archiemedean fuzzy metric space introduced by Mihet [6] and proved fixed point theorem under fuzzy $\psi$-contractive mapping in fuzzy metric space. Vetro [10] got some Common fixed point results for a pair of generalization contractive type mappings.

In this paper, we introduce the concept of intuitionistic $(\phi, \psi)$-contractive mappings and prove some a common fixed point theorems in intuitionistic fuzzy metric space under $(\phi, \psi)$-contractive mappings and weakly commuting intuitionistic fuzzy metric space.

\footnotetext{
*Corresponding author

Email addresses: donia_1000@yahoo.com (H. M. Abu-Donia), h_a_atia@hotmail.com (H. A. Atia), omnia.khater1693@yahoo.com (Omnia M. A. Khater)

doi: $10.22436 /$ jnsa.013.06.03
}

Received: 2020-01-19 Revised: 2020-02-05

Accepted: 2020-02-15 


\section{Preliminaries}

Definition $2.1([1])$. A binary operation $*:[0,1] \times[0,1] \longrightarrow[0,1]$ is continuous t-norm if $*$ satisfies the following conditions:

(i) $*$ is commutative and associative;

(ii) $*$ is continuous;

(iii) $a * 1=a$ for all $a \in[0,1]$;

(iv) $a * b \leqslant c * d$ whenever $a \leqslant c, b \leqslant d$ and $a, b, c, d \in[0,1]$.

For examples of t-norms we have

- $a * b=\min \{a, b\}$

- $a * b=a . b$;

- $a * b=\max \{0, a+b-1\}$;

- $a * b= \begin{cases}\min \{a, b\}, & \text { if } a+b>1, \\ 0, & \text { otherwise. }\end{cases}$

Definition 2.2 ([1]). A binary operation $\diamond:[0,1] \times[0,1] \longrightarrow[0,1]$ is continuous t-conorm if $\diamond$ satisfies the following conditions:

(i) $\diamond$ is commutative and associative;

(ii) $\diamond$ is continuous;

(iii) $a \diamond 1=a$ for all $a \in[0,1]$;

(iv) $a \diamond b \leqslant c \diamond d$ whenever $a \leqslant c, b \leqslant d$ and $a, b, c, d \in[0,1]$.

For examples of $\mathrm{t}$-conorms we have

- $a \diamond b=\max \{a, b\}$

- $a \diamond b=a+b-a . b$

- $a \diamond b=\min \{a+b, 1\}$

- $a \diamond b= \begin{cases}\max \{a, b\}, & \text { if } a+b<1, \\ 1, & \text { otherwise. }\end{cases}$

Definition $2.3([1])$. Let $(X, M, N, *, \diamond)$ is said to be an intuitionistic fuzzy metric space if $X$ is an arbitrary set, $*$ is a continuous t-norm, $\diamond$ is a continuous t-conorm and $M, N$ are fuzzy sets on $X \times X \times[0, \infty)$ satisfying the following conditions:

(i) $M(x, y, t)+N(x, y, t) \leqslant 1$ for all $x, y \in X$ and $t>0$;

(ii) $M(x, y, 0)=0$ for all $x, y \in X$;

(iii) $M(x, y, t)=1$ for all $x, y \in X$ and $t>0$ if and only if $x=y$;

(iv) $M(x, y, t)=M(y, x, t)$ for all $x, y \in X$ and $t>0$;

(v) $M(x, y, t) * M(y, z, s) \leqslant M(x, z, t+s)$ for all $x, y, z \in X$ and $t, s>0$;

(vi) $M(x, y,):.[0, \infty) \longrightarrow[0,1]$ is left continuous for all $x, y, \in X$;

(vii) $\lim _{t \rightarrow \infty} M(x, y, t)=1$ for all $x, y \in X$ and $t>0$;

(vii) $N(x, y, 0)=1$ for all $x, y \in X$;

(viii) $N(x, y, t)=0$ for all $x, y \in X$ and $t>0$ if and only if $x=y$;

(ix) $N(x, y, t)=N(y, x, t)$ for all $x, y \in X$ and $t>0$;

(x) $N(x, y, t) \diamond N(y, z, s) \geqslant N(x, z, t+s)$ for all $x, y, z \in X$ and $t, s>0$;

(xi) $N(x, y,):.[0, \infty) \longrightarrow[0,1]$ is right continuous for all $x, y, \in X$;

(xii) $\lim _{t \rightarrow \infty} N(x, y, t)=0$ for all $x, y \in X$ and $t>0$. 
Then $(M, N)$ is called an intuitionistic fuzzy metric space on $X$. The functions $M(x, y, t)$ and $N(x, y, t)$ denote the degree of nearness and the degree of non-nearness between $x$ and $y$ w.r.t. $t$ respectively.

Definition 2.4. Let $(X, M, N, *, \diamond)$ be an intuitionistic fuzzy metric space. Then a sequence $\left\{x_{n}\right\}$ in $X$ is said to be convergent to a point $x \in X$ for all $t>0, \lim _{n \rightarrow \infty} M\left(x_{n}, x, t\right)=1$ and $N\left(x_{n}, x, t\right)=0$.

Definition 2.5. Let $(X, M, N, *, \diamond)$ be an intuitionistic fuzzy metric space. Then a sequence $\left\{x_{n}\right\}$ in $X$ is said to be Cauchy sequence if, for all $t>0$ and $p>0, \lim _{n \rightarrow \infty} M\left(x_{n+p}, x_{n}, t\right)=1$ and $N\left(x_{n+p}, x_{n}, t\right)=0$.

Definition 2.6. An intuitionistic fuzzy metric space $(X, M, N, *, \diamond)$ is said to be complete if and only if every Cauchy sequence in $X$ is convergent.

Example 2.7. Let $X=\left\{\frac{1}{n} ; n=1,2,3, \ldots\right\} \bigcup\{0\}$ and let $*$ be the continuous t-norm and $\diamond$ be the continuous $\mathrm{t}$-conorm defined by $\mathrm{a} * \mathrm{~b}=\mathrm{ab}$ and $\mathrm{a} \diamond \mathrm{b}=\min \{1, \mathrm{a}+\mathrm{b}\}$ for all $\mathrm{a}, \mathrm{b} \in[0,1]$ for each $\mathrm{x}, \mathrm{y} \in \mathrm{X}$ and $\mathrm{t}>0$, define $(M, N)$ by

$$
M(x, y, t)=\left\{\begin{array}{ll}
0, & t=0, \\
\frac{t}{t+d(x, y)}, & t>0,
\end{array} \quad N(x, y, t)= \begin{cases}1, & t=0, \\
\frac{d(x, y)}{t+d(x, y)}, & t>0 .\end{cases}\right.
$$

Then $(X, M, N, *, \diamond)$ is an intuitionistic fuzzy metric space.

Definition 2.8. The class of all $\phi$ - functions will be denoted by $\Phi$ if $\phi:(0,1] \longrightarrow(0,1]$, is continuous non decreasing function, s.t. $\lim _{\mathrm{t} \rightarrow 0} \phi(t)=0$, and $\phi(1)=1$.

Definition 2.9. The class of all $\psi$ - functions will be denoted by $\psi$ if $\psi:[0, \infty) \longrightarrow[0, \infty)$, is continuous non decreasing function, s.t. $\lim _{\mathrm{t} \longrightarrow \infty} \psi(\mathrm{t})=\infty$, and $\psi(0)=0$.

Definition 2.10 ([8]). A pair of self mappings $(A, B)$ of an intuitionistic fuzzy metric space $(X, M, N, *, \diamond)$ is said to be weakly commuting if $M(A B x, B A x, t) \geqslant M(A x, B x, t), N(A B x, B A x, t) \leqslant N(A x, B x, t)$ for all $x \in X$ and $t>0$.

Definition 2.11. Let $(X, M, N, *, \diamond)$ be an intuitionistic fuzzy metric space and $\psi \in \Psi$. A mapping $A$ : $\mathrm{X} \longrightarrow \mathrm{X}$ is called $(\phi, \psi)$-contractive mapping if the following implication takes place:

$$
\begin{gathered}
M(x, y, t)>0 \Longrightarrow M(A(x), A(y), t) \geqslant \phi(M(x, y, t)), \\
N(x, y, t)<1 \Longrightarrow N(A(x), B(y), t) \leqslant \psi(N(x, y, t)) .
\end{gathered}
$$

Lemma 2.12. If $\phi \in \Phi$, then $\lim _{n \rightarrow \infty} \phi^{n}(t)=1$ for all $\mathrm{t} \in(0,1)$.

Lemma 2.13. If $\psi \in \Psi$, then $\lim _{n \rightarrow \infty} \psi^{n}(t)=0$ for all $t \in(0,1)$.

Example 2.14. Let $X=[0, \infty), a * b=\min \{a, b\}, a \diamond b=\max \{a, b\} \forall a, b \in[0,1]$ and define

$$
M(x, y, t)=\left\{\begin{array}{l}
0, t \leqslant d(x, y), \\
1, \quad t>d(x, y),
\end{array} \quad N(x, y, t)=\left\{\begin{array}{l}
1, t \leqslant d(x, y), \\
0, \quad t<d(x, y),
\end{array}\right.\right.
$$

for all $x, y \in X$ and $t>0$. Then $(X, M, N, *, \diamond)$ is an intuitionistic fuzzy metric space.

Let $\phi$ be a mapping in $\Phi$. Since $\phi(1)=1$,

$$
M(x, y, t)>0 \Longrightarrow M(x, y, t)=1 \Longrightarrow \phi(M(x, y, t))=1,
$$

and let $\psi$ be a mapping in $\Psi$. Since $\psi(0)=0$,

$$
N(x, y, t)<1 \Longrightarrow N(x, y, t)=0 \Longrightarrow \psi(N(x, y, t))=0,
$$

therefore every mapping $A: X \longrightarrow X$ is an intuitionistic fuzzy $(\phi, \psi)$-contractive mapping.

Definition 2.15. Let $(X, M, N, *, \diamond)$ be an intuitionistic fuzzy metric space and let $(A, B)$ is a pair of $(\phi, \psi)$ contractive mapping if there exist $\psi \in \Psi$ such that for every $x, y \in X$ and $t \in(0,1)$ with

$$
\begin{gathered}
M(x, y, t)>0 \Longrightarrow M(A(x), B(y), t) \geqslant \phi(\min \{M(x, y, t), M(A(x), x, t), M(y, B(y), t)\}), \\
N(x, y, t)<1 \Longrightarrow N(A(x), B(y), t) \leqslant \psi(\max \{N(x, y, t), N(A(x), x, t), N(y, B(y), t)\}) .
\end{gathered}
$$




\section{Main results}

Theorem 3.1. Let $A$ and $B$ be self mappings of a complete intuitionistic fuzzy metric space $(X, M, N, *, \diamond)$ such that $\mathrm{a} * \mathrm{~b}=\min \{\mathrm{a}, \mathrm{b}\}$ and $\mathrm{a} \diamond \mathrm{b}=\max \{\mathrm{a}, \mathrm{b}\}$ for all $\mathrm{a}, \mathrm{b} \in \mathrm{X}$. Assume $\mathrm{M}\left(\mathrm{x}_{\mathrm{o}}, \mathrm{A}\left(\mathrm{x}_{\mathrm{o}}\right), \mathrm{t}\right)>0$ and $\mathrm{N}\left(\mathrm{x}_{\mathrm{o}}, \mathrm{A}\left(\mathrm{x}_{\mathrm{o}}\right), \mathrm{t}\right)<1$ for all $\mathrm{x}_{\mathrm{o}} \in \mathrm{X}, \forall \mathrm{t}>0$, let $\mathrm{A}, \mathrm{B}$ be self mappings of $\mathrm{X}$ satisfying the following conditions:

(1) $A, B$ are two continuous and intuitionistic $\psi$-contractive mappings;

(2)

$$
\begin{aligned}
& M(A(x), B(y), t) \geqslant \phi(\min \{M(x, y, t), M(A(x), x, t), M(y, B(y), t)\}), \\
& N(A(x), B(y), t) \leqslant \psi(\max \{N(x, y, t), N(A(x), x, t), N(y, B(y), t)\})
\end{aligned}
$$

for all $\mathrm{x}, \mathrm{y} \in \mathrm{X}$ and $\mathrm{t}>0$;

(3) $\left\{x_{n}\right\}$ is $(A, B)$-sequence of initial point $x_{0}$.

Then $\mathrm{A}$ and $\mathrm{B}$ have a unique common fixed point in $\mathrm{X}$.

Proof. $\left\{x_{n}\right\}$ is $(A, B)$-sequence of initial point $x_{0}$. Then $x_{0} \in X$ and define the sequence $\left\{x_{n}\right\}$ by $x_{1}=$ $A\left(x_{0}\right), x_{2}=B\left(x_{1}\right), \ldots, x_{2 n+1}=A\left(x_{2 n}\right), x_{2 n+2}=B\left(x_{2 n+1}\right)$, from $M\left(x_{0}, A\left(x_{0}\right), t\right)=M\left(x_{0}, x_{1}, t\right)>0$ and $N\left(x_{0}, A\left(x_{0}\right), t\right)=N\left(x_{0}, x_{1}, t\right)<1$ it follows that,

$$
\begin{aligned}
M\left(x_{2}, x_{1}, t\right) & =M\left(A\left(x_{0}\right), B\left(x_{1}\right), t\right) \\
& \geqslant \phi\left(\min \left\{M\left(x_{0}, x_{1}, t\right), M\left(A\left(x_{0}\right), x_{0}, t\right), M\left(x_{1}, B\left(x_{1}\right), t\right)\right\}\right) \\
& \geqslant\left(M\left(x_{0}, x_{1}, t\right)\right)>0, \\
N\left(x_{2}, x_{1}, t\right) & =N\left(A\left(x_{0}\right), B\left(x_{1}\right), t\right) \\
& \leqslant \psi\left(\max \left\{N\left(x_{0}, x_{1}, t\right), N\left(A\left(x_{0}\right), x_{0}, t\right), N\left(x_{1}, B\left(x_{1}\right), t\right)\right\}\right) \\
& \leqslant\left(N\left(x_{0}, x_{1}, t\right)\right)<1, \\
M\left(x_{3}, x_{2}, t\right) & =M\left(A\left(x_{2}\right), B\left(x_{1}\right), t\right) \\
& \geqslant \phi\left(\min \left\{M\left(x_{2}, x_{1}, t\right), M\left(A\left(x_{2}\right), x_{2}, t\right), M\left(x_{1}, B\left(x_{1}\right), t\right)\right\}\right) \\
& \geqslant \phi\left(M\left(x_{2}, x_{1}, t\right)\right) \\
& \geqslant \phi^{2}\left(M\left(x_{0}, x_{1}, t\right)\right)>0 \\
N\left(x_{3}, x_{2}, t\right) & =N\left(A\left(x_{2}\right), B\left(x_{1}\right), t\right) \\
& \leqslant \psi\left(\max \left\{N\left(x_{2}, x_{1}, t\right), N\left(A\left(x_{2}\right), x_{2}, t\right), N\left(x_{1}, B\left(x_{1}\right), t\right)\right\}\right) \\
& \leqslant \psi\left(N\left(x_{2}, x_{1}, t\right)\right) \\
& \leqslant \psi\left(N\left(x_{0}, x_{1}, t\right)\right)<1 .
\end{aligned}
$$

Generally, for each $n$, we get $M\left(x_{n+1}, x_{n}, t\right) \geqslant \phi^{n}\left(M\left(x_{0}, x_{1}, t\right)\right)>0$ and $N\left(x_{n+1}, x_{n}, t\right) \leqslant \psi^{n}\left(N\left(x_{0}, x_{1}, t\right)\right)<$ 1. By Lemmas 2.12 and 2.13 as $n \longrightarrow \infty$ we get $\lim _{n \rightarrow \infty} M\left(x_{n+1}, x_{n}, t\right)=1, \lim _{n \rightarrow \infty} N\left(x_{n+1}, x_{n}, t\right)=0$. Then $\left\{x_{n}\right\}$ is a Cauchy. Since $X$ is Complete, then there exists $x \in X$ such that $x_{n} \longrightarrow x$, for all $t>0$.

$$
\begin{aligned}
& M\left(x_{2 n+1}, A(x), t\right)=M\left(A\left(x_{2 n}\right), A(x), t\right) \geqslant \phi\left(M\left(x_{2 n}, x, t\right)\right), \\
& N\left(x_{2 n+1}, A(x), t\right)=N\left(A\left(x_{2 n}\right), A(x), t\right) \leqslant \psi\left(N\left(x_{2 n}, x, t\right)\right) .
\end{aligned}
$$

Since $A$ is continuous, letting $n \longrightarrow \infty$ it follows, $M(x, A(x), t) \geqslant \phi(1)=1$ and $N(x, A(x), t) \leqslant \psi(0)=0$, hence $A(x)=x$. Similarity we get $B(x)=x$ and $x$ is common fixed point of $A$ and $B$. Assume that $x, y \in X$ are two common fixed points of $A$ and $B$. If $x \neq y$, there exists $t>0$, such that $0<M(x, y, t)<1$ and $0>N(x, y, t)>1$. Then

$$
\begin{aligned}
M(x, y, t) & =M(A(x), B(y), t) \\
& \geqslant \phi(\min \{M(x, y, t), M(A(x), x, t), M(y, B(y), t)\})=\phi(M(x, y, t))>M(x, y, t),
\end{aligned}
$$

$N(x, y, t)=N(A(x), B(y), t) \leqslant \psi(\max \{N(x, y, t), N(A(x), x, t), N(y, B(y), t)\})=\psi(N(x, y, t))<N(x, y, t)$.

This is a contradiction, therefore $x=y$. 
Theorem 3.2. Let $A, B$ and $T$ be self mappings of a Complete intuitionistic fuzzy metric space $(X, M, N, *, \diamond)$ such that $\mathrm{a} * \mathrm{~b}=\min \{\mathrm{a}, \mathrm{b}\}, \mathrm{a} \diamond \mathrm{b}=\max \{\mathrm{a}, \mathrm{b}\}$ for all $\mathrm{a}, \mathrm{b} \in \mathrm{X}$, let $\mathrm{A}, \mathrm{B}$ and $\mathrm{T}$ be self mappings of $\mathrm{X}$ satisfying the following conditions:

(1) $\mathrm{A}$ is intuitionistic $\psi$-contractive mapping and $\mathrm{B}, \mathrm{T}$ are two continuous mappings;

(2) $\mathrm{A}(\mathrm{x}) \subset \mathrm{B}(\mathrm{x}) \cap \mathrm{T}(\mathrm{x})$ and $\{\mathrm{A}, \mathrm{B}\},\{\mathrm{A}, \mathrm{T}\}$ are weakly commuting;

(3)

$$
\begin{aligned}
& M(A x, A y, t) \geqslant \phi(\min \{M(B x, T y, t), M(B x, A x, t), M(B x, A y, t), M(T y, A y, t)\}), \\
& N(A x, A y, t) \leqslant \psi(\max \{N(B x, T y, t), N(B x, A x, t), N(B x, A y, t), N(T y, A y, t)\}),
\end{aligned}
$$

for all $\mathrm{x}, \mathrm{y} \in \mathrm{X}$ and $\mathrm{t}>0$.

Then $\mathrm{A}, \mathrm{B}$ and $\mathrm{T}$ have a unique Common fixed point in $\mathrm{X}$.

Proof. Let $x_{0} \in X$ be an arbitrary point. Then there exist a point $x_{1} \in X$ such that $A x_{0}=B x_{1}$. Since $A(x) \subset T(x)$, then there exists a point $x_{2} \in X$ such that $A x_{1}=T x_{2}$. In general, we get a sequence $\left\{y_{n}\right\}$ recursively as $y_{n}=B x_{n+1}=A x_{n}$ and $y_{n+1}=T x_{n+2}=A x_{n+1}, n \in N$.

Let $M_{n}=M\left(y_{n+1}, y_{n}, t\right)=M\left(A x_{n+1}, A x_{n}, t\right)$ and $N_{n}=N\left(y_{n+1}, y_{n}, t\right)=N\left(A x_{n+1}, A x_{n}, t\right)$ and $M\left(y_{0}, y_{1}, t\right)>0, N\left(y_{0}, y_{1}, t\right)<1$. Then

$M_{n+1}=M\left(y_{n+2}, y_{n+1}, t\right)=M\left(A x_{n+2}, A x_{n+1}, t\right)$ and $\quad N_{n+1}=N\left(y_{n+2}, y_{n+1}, t\right)=N\left(A x_{n+2}, A x_{n+1}, t\right)$.

Using inequality (3), we get,

$$
\begin{aligned}
M_{n+1} & =M\left(A x_{n+2}, A x_{n+1}, t\right) \\
& \geqslant \phi\left(\min \left\{M\left(B x_{n+2}, T x_{n+1}, t\right), M\left(B x_{n+2}, A x_{n+2}, t\right), M\left(B x_{n+2}, A x_{n+1}, t\right), M\left(T x_{n+1}, A x_{n+1}, t\right)\right\}\right), \\
& =\phi\left(\min \left\{M\left(A x_{n+1}, A x_{n}, t\right), M\left(A x_{n+1}, A x_{n+2}, t\right), M\left(A x_{n+1}, A x_{n+1}, t\right), M\left(A x_{n}, A x_{n+1}, t\right)\right\}\right) \\
& =\phi\left(\min \left\{M_{n}, M_{n+1}, 1, M n\right\}\right), \\
N_{n+1} & =N\left(A x_{n+2}, A x_{n+1}, t\right) \\
& \leqslant \psi\left(\max \left\{N\left(B x_{n+2}, T x_{n+1}, t\right), N\left(B x_{n+2}, A x_{n+2}, t\right), N\left(B x_{n+2}, A x_{n+1}, t\right), N\left(T x_{n+1}, A x_{n+1}, t\right)\right\}\right) \\
& =\psi\left(\max \left\{N\left(A x_{n+1}, A x_{n}, t\right), N\left(A x_{n+1}, A x_{n+2}, t\right), N\left(A x_{n+1}, A x_{n+1}, t\right), N\left(A x_{n}, A x_{n+1}, t\right)\right\}\right) \\
& =\psi\left(\max \left\{N_{n}, N_{n+1}, 0, N_{n}\right\}\right) .
\end{aligned}
$$

If $M_{n}>M_{n+1}$ and $N_{n}<N_{n+1}$, then by definition of $(\phi, \psi)$-we have

$$
M_{n+1} \geqslant \phi\left(M_{n+1}\right)>M_{n+1}, \quad N_{n+1} \leqslant \psi\left(N_{n+1}\right)<N_{n+1} .
$$

This is a contradiction, so,

$$
M_{n+1} \geqslant \phi\left(M_{n}\right), \quad N_{n+1} \leqslant \psi\left(N_{n}\right) .
$$

We get, $M\left(y_{n+2}, y_{n+1}, t\right) \geqslant \phi\left(M\left(y_{n+1}, y_{n}, t\right)\right)$ and $N\left(y_{n+2}, y_{n+1}, t\right) \leqslant \psi\left(N\left(y_{n+1}, y_{n}, t\right)\right), \quad \forall n \in N, t>0$. Hence, repeating this inequality $\mathrm{n}$ times we obtain,

$$
M\left(y_{n}, y_{n+1}, t\right) \geqslant \phi^{n}\left(M\left(y_{0}, y_{1}, t\right)\right), \quad N\left(y_{n}, y_{n+1}, t\right) \leqslant \psi^{n}\left(N\left(y_{0}, y_{1}, t\right) .\right.
$$

Letting $n \longrightarrow \infty$, we get $\lim _{n \rightarrow \infty} M\left(y_{n}, y_{n+1}, t\right)=1$ and $\lim _{n \rightarrow \infty} N\left(y_{n}, y_{n+1}, t\right)=0$. Then $\left\{y_{n}\right\}$ is a Cauchy. Since $X$ is Complete, then there exists $z \in X$ such that $y_{n} \longrightarrow z$. Hence $\left(A x_{n}\right) \longrightarrow z \in X$. Since $A$ is intuitionistic $(\phi, \psi)$-contractive mapping,

$$
M\left(y_{n}, A z, t\right)=M\left(A x_{n}, A z, t\right) \geqslant \phi\left(M\left(x_{n}, z, t\right)\right), \quad N\left(y_{n}, A z, t\right)=N\left(A x_{n}, A z, t\right) \leqslant \psi\left(N\left(x_{n}, z, t\right)\right) .
$$


By taking the limit as $n \longrightarrow \infty$ we obtain,

$$
\mathrm{M}(z, \mathrm{~A} z, \mathrm{t}) \geqslant \phi(1)=1, \quad \mathrm{~N}(z, \mathrm{Az}, \mathrm{t}) \leqslant \psi(0)=0,
$$

hence $A z=z$.Since $\left(A x_{n}\right) \longrightarrow z \in X$, hence the sub-sequences $\left\{B x_{n}\right\}$ and $\left\{T x_{n}\right\}$ of $\left\{A x_{n}\right\}$ have the same limit. Since $B$ is continuous, in this case we have $B A x_{n} \longrightarrow B z, B B x_{n} \longrightarrow B z$. Also $(A, B)$ is weakly commuting, we have $A B x_{n} \longrightarrow B z$. Let $x=B x_{n}, y=x_{n}$ in (3), we get

$$
\begin{aligned}
& M\left(A B x_{n}, A x_{n}, t\right) \geqslant \phi\left(\min \left\{M\left(B B x_{n}, T x_{n}, t\right), M\left(B B x_{n}, A B x_{n}, t\right), M\left(B B x_{n}, A x_{n}, t\right), M\left(T x_{n}, A x_{n}, t\right)\right\}\right), \\
& N\left(A B x_{n}, A x_{n}, t\right) \leqslant \psi\left(\max \left\{N\left(B B x_{n}, T x_{n}, t\right), N\left(B B x_{n}, A B x_{n}, t\right), N\left(B B x_{n}, A x_{n}, t\right), N\left(T x_{n}, A x_{n}, t\right)\right\}\right) .
\end{aligned}
$$

Taking limit $\mathrm{n} \longrightarrow \infty$ we get,

$$
\begin{aligned}
M(B z, z, t) & \geqslant \phi(\min \{M(B z, z, t), M(B z, B z, t), M(B z, z, t), M(z, z, t)\}) \\
& =\phi(\min \{M(B z, z, t), 1, M(B z, z, t), 1\}) \\
& =\phi(M(B z, z, t))>M(B z, z, t) \\
N(B z, z, t) & \leqslant \psi(\max \{N(B z, z, t), N(B z, B z, t), N(B z, z, t), N(z, z, t)\}) \\
& =\psi(\max \{N(B z, z, t), 0, N(B z, z, t), 0\}) \\
& =\psi(N(B z, z, t))<N(B z, z, t) .
\end{aligned}
$$

So we get, $B z=z$. Since $T$ is continuous, in this case we have $T T x_{n} \longrightarrow T z, T A x_{n} \longrightarrow T z$. Also $(A, T)$ is weakly commuting, we have $A T x_{n} \longrightarrow T z$, let $x=x_{n}, y=T x_{n}$ in (3), we get,

$$
\begin{aligned}
& M\left(A x_{n}, A T x_{n}, t\right) \geqslant \phi\left(\min \left\{M\left(B x_{n}, T T x_{n}, t\right), M\left(B x_{n}, A x_{n}, t\right), M\left(B x_{n}, A T x_{n}, t\right), M\left(T T x_{n}, A T x_{n}, t\right)\right\}\right), \\
& N\left(A x_{n}, A T x_{n}, t\right) \leqslant \psi\left(\max \left\{N\left(B x_{n}, T T x_{n}, t\right), N\left(B x_{n}, A x_{n}, t\right), N\left(B x_{n}, A T x_{n}, t\right), N\left(T T x_{n}, A T x_{n}, t\right)\right\}\right) .
\end{aligned}
$$

Taking limit as $n \longrightarrow \infty$,

$$
\begin{aligned}
\mathrm{M}(z, \mathrm{~T} z, \mathrm{t}) & \geqslant \phi(\min \{\mathrm{M}(z, \mathrm{~T} z, \mathrm{t}), M(z, z, \mathrm{t}), \mathrm{M}(z, \mathrm{~T} z, \mathrm{t}), \mathrm{M}(\mathrm{T} z, \mathrm{~T} z, \mathrm{t})\}) \\
& =\phi(\min \{\mathrm{M}(z, \mathrm{~T} z, \mathrm{t}), 1, \mathrm{M}(z, \mathrm{~T} z, \mathrm{t}), 1\}) \\
& =\phi(M(z, \mathrm{~T} z, \mathrm{t}))>M(z, \mathrm{~T} z, \mathrm{t}) \\
\mathrm{N}(z, \mathrm{~T} z, \mathrm{t}) & \leqslant \psi(\max \{\mathrm{N}(z, \mathrm{~T} z, \mathrm{t}), \mathrm{N}(z, z, \mathrm{t}), \mathrm{N}(z, \mathrm{~T} z, \mathrm{t}), \mathrm{N}(\mathrm{T} z, \mathrm{~T} z, \mathrm{t})\}) \\
& =\psi(\max \{\mathrm{N}(z, \mathrm{~T} z, \mathrm{t}), 0, \mathrm{~N}(z, \mathrm{~T} z, \mathrm{t}), 0\}) \\
& =\psi(\mathrm{N}(z, \mathrm{~T} z, \mathrm{t}))<\mathrm{N}(z, \mathrm{~T} z, \mathrm{t}) .
\end{aligned}
$$

So, we get $T z=z$. Thus, we have $A z=B z=T z=z$, then $z$ is a common fixed point of $A$, $B$, and $T$. Now we prove the uniqueness of the common fixed points of $A, B$, and T. Let $v$ be another common fixed point of $A, B$, and $T$. Then $A v=B v=T v=v$. Take $x=z, y=v$ in(3), we get,

$$
\begin{aligned}
& M(z, v, t) \geqslant \phi(\min \{M(B z, T v, t), M(B z, A z, t), M(B z, A v, t), M(T v, A z, t)\})=\phi(M(z, v, t))>M(z, v, t), \\
& N(z, v, t) \leqslant \psi(\max \{N(B z, T v, t), N(B z, A z, t), N(B z, A v, t), N(T v, A z, t)\})=\psi(N(z, v, t))<N(z, v, t) .
\end{aligned}
$$

We get $z=v$. Therefore $z$ is a unique common fixed point of $A, B$, and $T$.

\section{References}

[1] C. Alaca, D. Turkoglu, C. Yildiz, Fixed points in intuitionistic fuzzy metric spaces, Chaos Solitons Fractals, 29 (2006), 1073-1078. 2.1, 2.2, 2.3

[2] K. T. Atanassov, Intuitionistic fuzzy sets, Physica-Verlag, Heidelberg, (1999). 1

[3] V. Gregori, J.-J. Minana, D. Miravet, Contractive sequences in fuzzy metric spaces, Fuzzy Sets and Systems, 379 (2020), 125-133. 1 
[4] G. Jungck, B. E. Rhoades, Fixed point for set valued functions without continuity, Indian J. Pure Appl. Math., 29 (1998), 227-238. 1

[5] I. Kramosil, J. Michálek, Fuzzy metric and statistical metric spaces, Kybernetika (Prague), 11 (1975), 336-344. 1

[6] D. Miheț, Fuzzy భ-contractive mappings in non-Archimedean fuzzy metric spaces, Fuzzy Sets and Systems, 159 (2008), 739-744. 1

[7] J. H. Park, Intuitionistic fuzzy metric spaces, Chaos Solitons Fractals, 22 (2004), 1039-1046. 1

[8] S. Sessa, On a weak commutativity condition of mappings in fixed point considerations, Publ. Inst. Math. (Beograd) (N.S.), 32 (1982), 149-153. 1, 2.10

[9] D. Turkoglu, C. Alaca, Y. J. Cho, C. Yildiz, Common fixed point theorems in intuitionistic fuzzy metric spaces, J. Appl. Math. Comput., 22 (2006), 411-424.

[10] C. Vetro, Fixed points in weak non-Archimedean fuzzy metric spaces, Fuzzy Sets and Systems, 162 (2011), 84-90. 1

[11] X. Wu, G. Chen, Answering an open question in fuzzy metric spaces, Fuzzy Sets and Systems, 2019 (2019), 4 pages. 1

[12] L. A. Zadeh, Fuzzy sets, Inf. Control, 8 (1965), 338-353. 1

[13] D. Zheng, P. Wang, Meir-Keeler theorems in fuzzy metric spaces, Fuzzy Sets and Systems, 370 (2019), 120-128. 1 